\title{
THE ASYMPTOTIC BEHAVIOR OF THE STABLE INITIAL MANIFOLDS OF A SYSTEM OF NONLINEAR DIFFERENTIAL EQUATIONS
}

\author{
BX \\ J. J. LEVIN
}

1. Introduction. In a previous paper, [5], we investigated the behavior as $\epsilon \rightarrow 0+$ of the solutions of the system of real nonlinear differential equations

$$
\frac{d x}{d t}=f(t, x, y, \epsilon), \quad \epsilon \frac{d y}{d t}=g(t, x, y, \epsilon)
$$

where $x$ and $y$ are vectors of $m$ and $n$ components respectively, which satisfy initial conditions (at $t=0$ ) close to those of a known solution, $x=p(t)$, $y=q(t) \in C^{\prime}(0 \leqq t \leqq T)$, of the system

$$
\frac{d x}{d t}=f(t, x, y, 0), \quad 0=g(t, x, y, 0) .
$$

Here we shall show that those solutions of (1.1) whose initial $y$ vector lies on a certain "stable" initial manifold, which depends on $\epsilon$ and the initial $x$ vector, are very well approximated for small $t$ and $\epsilon$ by the corresponding solutions of a boundary layer equation, in which the initial $x$ vector enters as a parameter, associated with (1.1).. Moreover, it will also be shown that as $\epsilon \rightarrow 0+$ the stable initial manifolds associated with (1.1) tend to the stable initial manifold associated with the boundary layer equation.

Problems of this nature, i.e., problems in which there is a system of differential equations possessing the property that the setting of a parameter equal to zero reduces the order of the system, have been treated under various hypotheses by several authors, e.g., $[2 ; 3 ; 4 ; 5 ; 6]$ and [7]. The same hypotheses as in [5] will be assumed here. We now state these hypotheses as well as the principal theorem of [5]; this will then serve to introduce the problem and the results of the present paper.

Let $f_{x}=f_{x}(t, x, y, \epsilon)$ be the matrix with $\partial f_{i} / \partial x_{j}$ in the $i$ th row and $j$ th column and let $f_{x}(t)$ be the matrix $f_{x}(t, p(t), q(t), 0)$. The matrices $f_{y}, g_{x}, g_{y}$ as well as $f_{y}(t), g_{x}(t), g_{y}(t)$ are similarly defined.

H1: $f, g, f_{x}, f_{y}, g_{x}, g_{y}$ are continuous in $(t, x, y, \epsilon)$ for $0 \leqq t \leqq T$ if $|x-p(t)|$ $+|y-q(t)|+\epsilon$ is sufficiently small.

$\mathrm{H} 2$ : There exists a real nonsingular matrix $P(t) \in C^{\prime}(0 \leqq t \leqq T)$ such that

Presented to the Society, August 24, 1956; received by the editors July 4, 1956. 


$$
P^{-1}(t) g_{y}(t) P(t)=\left(\begin{array}{cc}
B(t) & 0 \\
0 & C(t)
\end{array}\right) \quad(0 \leqq t \leqq T)
$$

where $B(t)$ is an $n_{1}$ by $n_{1}$ matrix each of whose characteristic roots has a negative real part for $0 \leqq t \leqq T$, and where $C(t)$ is an $n_{2}$ by $n_{2}$ matrix each of whose characteristic roots has a positive real part for $0 \leqq t \leqq T$. Thus $n=n_{1}+n_{2}$.

H3: $g_{y}^{-1}(t) g_{x}(t) \in C^{\prime}(0 \leqq t \leqq T)$.

TheOREM 1. There exists a positive constant $\gamma_{1}$ which is independent of $\epsilon$ and an $n_{1}$ dimensional manifold, $S(a, \epsilon)$, in y space which depends continuously on $(a, \epsilon)$ for $|a| \leqq \gamma_{1}, 0<\epsilon \leqq \gamma_{1}$, such that if the initial vector for $x$ is $p(0)+a$ and for $y$ lies on $S(a, \epsilon)$ then the solution $x=\bar{x}(t, a, b, \epsilon), y=\bar{y}(t, a, b, \epsilon)$ of (1.1) (where $b=\left\{b_{1}, \cdots, b_{n_{1}}\right\}$ are curvilinear coordinates on $\left.S(a, \epsilon)\right)$ is unique, exists over the interval $0 \leqq t \leqq T$, and satisfies the inequalities

$$
\begin{aligned}
& |\bar{x}(t, a, b, \epsilon)-p(t)| \leqq K(|a|+\epsilon|b|+\omega(\epsilon)) \quad(0 \leqq t \leqq T), \\
& |\bar{y}(t, a, b, \epsilon)-q(t)| \leqq K\left(|a|+\epsilon|b|+|b| e^{-\sigma t / 2 \epsilon}+\omega(\epsilon)\right)
\end{aligned}
$$

where $K$ and $\sigma$ are positive constants independent of $\epsilon$, and where $\omega(\epsilon)$ is a continuous function of $\epsilon$ for $0 \leqq \epsilon \leqq \gamma_{1}$ with $\omega(0)=0$.

The manifold $S(a, \epsilon)$ is defined by

$$
S(a, \epsilon)=\left\{y \mid y=q(0)+P(0)\left(\begin{array}{c}
b \\
z(a, b, \epsilon)
\end{array}\right)-g_{y}^{-1}(0) g_{x}(0) a\right\}
$$

where $b=\left\{b_{1}, \cdots, b_{n_{1}}\right\}$ (as above), and where $z=\left\{z_{1}, \cdots, z_{n_{2}}\right\}$ is a continuous vector function of $(a, b, \epsilon)$ for $|a| \leqq \gamma_{1},|b| \leqq \gamma_{1}, 0<\epsilon \leqq \gamma_{1}$. The $S(a, \epsilon)$ are the stable initial manifolds referred to above.

In [5] it was also shown that for any $\epsilon$ in $0<\epsilon \leqq \gamma_{1}$ the initial $y$ vector does not have to lie precisely on $S(a, \epsilon)$ for the solution of (1.1) to exist over the interval $0 \leqq t \leqq T$. On the other hand, it was shown that the initial $y$ vector corresponding to any solution of (1.1) which does stay for $0 \leqq t \leqq T$ inside a sufficiently small tube $(|x-p(t)| \leqq \lambda,|y-q(t)| \leqq \lambda$ where $\lambda>0$ and independent of $\epsilon$ ) surrounding the solution $x=p(t), y=q(t)$ of (1.2) must be "close" to $S(a, \epsilon)$, and that, in fact, this "closeness" becomes exponentially small as $\epsilon \rightarrow 0+$.

The behavior of the manifolds $S(a, \epsilon)$ as $\epsilon \rightarrow 0+$, which was not investigated in [5] and whose analysis seems to require essentially different techniques from those employed there, will be obtained here as a by-product of a more general result (Theorem 3) concerning the following boundary layer equation (or, more accurately, system) related to (1.1):

$$
\epsilon \frac{d y^{*}}{d t}=g\left(0, p(0)+a, y^{*}, 0\right) .
$$


The motivation for considering (1.5) in order to investigate the solutions of (1.1) for small $t$ and $\epsilon$ is evident when one observes that $g$ is continuous in its arguments and that $d \bar{x} / d t$ is bounded. The latter follows from (1.3) and the first equation of (1.1).

We remark at the outset that the constant $K$ and the function $\omega(\epsilon)$ will be used generically, i.e., they may not be the same in each inequality, but they will have the same properties as in (1.3). However, it may be assumed without loss of generality that the constant $\gamma_{1}$ is the same throughout-as well as the constant $\gamma_{2}$, which first appears in Theorem 2 .

Setting $\tau=t / \epsilon$ in (1.5) yields

$$
\frac{d y^{*}}{d \tau}=g\left(0, p(0)+a, y^{*}, 0\right) .
$$

In $\S 3$ the proof of the following stability theorem for (1.6) will be indicated:

THEOREM 2. There exists a constant $\gamma_{2}>0$ and an $n_{1}$ dimensional manifold, $S^{*}(a)$, in $y^{*}$ space which depends continuously on a for $|a| \leqq \gamma_{1}$, such that if the initial vector for $y^{*}$ lies on $S^{*}(a)$ then the solution $y^{*}=\bar{y}^{*}(\tau, a, b)$ of $(1.6)$ (where $b=\left\{b_{1}, \cdots, b_{n_{1}}\right\}$ are curvilinear coordinates on $\left.S^{*}(a)\right)$ is unique, exists for $0 \leqq \tau<\infty$, and satisfies the inequality

$$
\left|\bar{y}^{*}(\tau, a, b)-q(0)\right| \leqq K\left(|a|+|b| e^{-\sigma \tau / 2}\right) \leqq \gamma_{2} \quad(0 \leqq \tau<\infty) .
$$

Furthermore, any solution, $y^{*}(\tau)$, of (1.6) however near $S^{*}(a)$ but not on $S^{*}(a)$ at $\tau=0$ cannot satisfy $\left|y^{*}(\tau)-q(0)\right| \leqq \gamma_{2}$ for $\tau \geqq 0$.

The manifold $S^{*}(a)$ is defined by

$$
S^{*}(a)=\left\{y^{*} \mid y^{*}=q(0)+P(0)\left(\begin{array}{c}
b \\
z^{*}(a, b)
\end{array}\right)-g_{y}^{-1}(0) g_{x}(0) a\right\}
$$

where $z^{*}$ has $n_{2}$ components and is a continuous function of $(a, b)$ for $|a| \leqq \gamma_{1}$, $|b| \leqq \gamma_{1}$. It is important to note that $S^{*}(a)$ does not depend on $\epsilon$. Clearly $\bar{y}^{*}(t / \epsilon, a, b)$ is a solution of (1.5).

In the following theorem, whose proof is given in $\S 4$, the solutions of (1.1) and (1.5) are related:

THEOREM 3. Given any $\alpha>0$ there exists $a \beta=\beta(\alpha)>0$ such that for $|a| \leqq \gamma_{1}$, $|b| \leqq \gamma_{1}$ (where $\gamma_{1}$ is independent of $\alpha$ )

$$
\begin{aligned}
|\bar{x}(t, a, b, \epsilon)-p(0)-a| & \leqq \alpha, \\
\left|\bar{y}(t, a, b, \epsilon)-\bar{y}^{*}(t / \epsilon, a, b)\right| & \leqq \alpha
\end{aligned}
$$

if $0 \leqq t \leqq \beta, 0<\epsilon \leqq \beta$, where $\bar{x}, \bar{y}$, and $\bar{y}^{*}$ are the same as in Theorems 1 and 2 .

From (1.9) we see that those solutions of the boundary layer equation which start on $S^{*}(a)$ do indeed yield good approximations for small $t$ and $\epsilon$ 
to those solutions of the system (1.1) with initial $y$ vector on $S(a, \epsilon)$. Moreover, upon setting $t=0$ in the second inequality of (1.9) and using (1.4) and (1.8) one easily has that $z(a, b, \epsilon) \rightarrow z^{*}(a, b)$ uniformly in $(a, b)$ as $\epsilon \rightarrow 0+$, and hence that $S(a, \epsilon) \rightarrow S^{*}(a)$ as $\epsilon \rightarrow 0+$. It may be mentioned, however, that while $S^{*}(a)$ is the limit of the stable initial manifolds for (1.1) it is in general not itself a stable initial manifold for (1.1). In particular, one can construct examples such that the solutions of (1.1) with initial $y$ vector on $S^{*}(a)$ are unbounded as $\epsilon \rightarrow 0+$.

2. Preliminaries. In this section we give that part of the machinery of [5] that is used in the proof of Theorem 3. The proofs of all unproven statements in this section may be found in [5].

Introducing the change of variables

$$
x=p(t)+\xi, \quad y=q(t)+P(t)\left(\begin{array}{l}
\eta \\
\zeta
\end{array}\right)-g_{y}^{-1}(t) g_{x}(t) \xi
$$

where $\eta$ and $\zeta$ have $n_{1}$ and $n_{2}$ components respectively, in (1.1) yields

$$
\begin{aligned}
\frac{d \xi}{d t} & =A(t) \xi+A_{1}(t) \eta+A_{2}(t) \zeta+H(t, \xi, \eta, \zeta, \epsilon) \\
\epsilon \frac{d \eta}{d t} & =B(t) \eta+R(t, \xi, \eta, \zeta, \epsilon), \\
\epsilon \frac{d \zeta}{d t} & =C(t) \zeta+S(t, \xi, \eta, \zeta, \epsilon)
\end{aligned}
$$

where

$$
A(t)=f_{x}(t)-f_{y}(t) g_{y}^{-1}(t) g_{x}(t), \quad A_{1}(t)=f_{y}(t) P_{1}(t), \quad A_{2}(t)=f_{y}(t) P_{2}(t)
$$

and where $P=\left(P_{1} P_{2}\right)-P_{1}$ and $P_{2}$ have $n_{1}$ and $n_{2}$ columns respectively. (The $S$ of (2.2) is, of course, different from the manifold $S(a, \epsilon)) . H, R$, and $S$ have the following properties which for brevity are stated only for $H$ : Given any $\delta>0$ there exists a $\gamma=\gamma(\delta)>0$ such that

$$
|H(t, \xi, \eta, \zeta, \epsilon)-H(t, \tilde{\xi}, \tilde{\eta}, \tilde{\zeta}, \epsilon)| \leqq \delta(|\xi-\tilde{\xi}|+|\eta-\tilde{\eta}|+|\zeta-\tilde{\zeta}|)
$$
for $0 \leqq t \leqq T$ if $|\xi|,|\tilde{\xi}|,|\eta|,|\tilde{\eta}|,|\zeta|,|\tilde{\zeta}|, \epsilon \leqq \gamma(\delta)$. Furthermore,

$$
|H(t, 0,0,0, \epsilon)| \leqq \omega(\epsilon) \quad(0 \leqq t \leqq T)
$$

if $\epsilon$ is sufficiently small.

Let $X(t), U(t, \epsilon), V(t, \epsilon)$ be fundamental matrices for

$$
\frac{d \xi}{d t}=A(t) \xi, \quad \epsilon \frac{d \eta}{d t}=B(t) \eta, \quad \epsilon \frac{d \zeta}{d t}=C(t) \zeta \quad(0 \leqq t \leqq T)
$$

respectively, and let 


$$
\begin{aligned}
\theta(t, s) & =X(t) X^{-1}(s), \\
\phi(t, s, \epsilon) & =U(t, \epsilon) U^{-1}(s, \epsilon) \quad(0 \leqq t, s \leqq T), \\
\psi(t, s, \epsilon) & =V(t, \epsilon) V^{-1}(s, \epsilon) .
\end{aligned}
$$

Then

$$
\begin{array}{rlrl}
|\theta(t, s)| & \leqq K & (0 \leqq t, s \leqq T), \\
|\phi(t, s, \epsilon)| \leqq K e^{-\sigma(t-s) / \epsilon} & (0 \leqq s \leqq t \leqq T), \\
|\psi(t, s, \epsilon)| \leqq K e^{-\sigma(s-t) / \epsilon} & (0 \leqq t \leqq s \leqq T)
\end{array}
$$

where $\sigma$ is the same as in (1.3).

There exist positive constants $\gamma_{1}$ and $\gamma_{2}$ which are independent of $\epsilon$ such that for $|a| \leqq \gamma_{1},|b| \leqq \gamma_{1}, 0<\epsilon \leqq \gamma_{1}$ the system of integral equations

$$
\begin{aligned}
& \xi(t, \epsilon)=\theta(t, 0) a+\int_{0}^{t} \theta(t, s)\left\{A_{1}(s) \eta(s, \epsilon)+A_{2}(s) \zeta(s, \epsilon)\right. \\
& +H(s, \xi(s, \epsilon), \eta(s, \epsilon), \zeta(s, \epsilon), \epsilon)\} d s \\
& \eta(t, \epsilon)=\phi(t, 0, \epsilon) b+\frac{1}{\epsilon} \int_{0}^{t} \phi(t, s, \epsilon) R(s, \xi(s, \epsilon), \eta(s, \epsilon), \zeta(s, \epsilon), \epsilon) d s, \\
& \zeta(t, \epsilon)=-\frac{1}{\epsilon} \int_{t}^{T} \psi(t, s, \epsilon) S(s, \xi(s, \epsilon), \eta(s, \epsilon), \zeta(s, \epsilon), \epsilon) d s
\end{aligned}
$$

has a unique solution, $\xi=\bar{\xi}(t, a, b, \epsilon), \eta=\bar{\eta}(t, a, b, \epsilon), \zeta=\bar{\zeta}(t, a, b, \epsilon)$, which satisfies the inequalities $|\xi(t, \epsilon)| \leqq \gamma_{2},|\eta(t, \epsilon)| \leqq \gamma_{2},|\zeta(t, \epsilon)| \leqq \gamma_{2}$. The functions $\bar{\xi}, \bar{\eta}, \bar{\xi}$ also satisfy the inequalities

$$
\begin{aligned}
& |\bar{\xi}(t, a, b, \epsilon)| \leqq K(|a|+\epsilon|b|+\omega(\epsilon)) \leqq \gamma_{2} \quad(0 \leqq t \leqq T), \\
& |\bar{\eta}(t, a, b, \epsilon)|,|\bar{\zeta}(t, a, b, \epsilon)| \leqq K\left(|a|+\epsilon|b|+|b| e^{-\sigma t / 2 \epsilon}+\omega(\epsilon)\right) \leqq \gamma_{2} .
\end{aligned}
$$

The solution $\bar{x}, \bar{y}$ of (1.1) referred to in Theorem 1 , is defined by setting $\xi=\bar{\xi}, \eta=\bar{\eta}, \zeta=\bar{\xi}$, in (2.1). The function $z(a, b, \epsilon)$ of (1.4) is defined by

$$
z(a, b, \epsilon)=-\frac{1}{\epsilon} \int_{0}^{T} \psi(0, s, \epsilon) S(s, \bar{\xi}(s, a, b, \epsilon), \bar{\eta}(s, a, b, \epsilon), \bar{\zeta}(s, a, b, \epsilon), \epsilon) d s .
$$

3. Proof of Theorem 2. Introducing the change of variables

$$
y^{*}=q(0)+P(0)\left(\begin{array}{l}
\eta^{*} \\
\zeta^{*}
\end{array}\right)-g_{y}^{-1}(0) g_{x}(0) a
$$

where $\eta^{*}$ and $\zeta^{*}$ have $n_{1}$ and $n_{2}$ components respectively, in (1.6) yields, after a little computation,

$$
\frac{d \eta^{*}}{d \tau}=B_{0} \eta^{*}+R\left(0, a, \eta^{*}, \zeta^{*}, 0\right), \quad \frac{d \zeta^{*}}{d \tau}=C_{0} \zeta^{*}+S\left(0, a, \eta^{*}, \zeta^{*}, 0\right)
$$


where $B_{0}=B(0), C_{0}=C(0)$ and where $R$ and $S$ are the same as in (2.2). We have the following lemma concerning (3.2):

LemMa. There exist positive constants $\gamma_{1}$ and $\gamma_{2}$ and an $n_{1}$ dimensional manifold, $S^{\prime}(a)$, in $\left\{\eta^{*}, \zeta^{*}\right\}$ space which depends continuously on a for $|a| \leqq \gamma_{1}$, such that if the initial vector for $\left\{\eta^{*}, \zeta^{*}\right\}$ lies on $S^{\prime}(a)$ then the solution $\eta^{*}$ $=\bar{\eta}^{*}(\tau, a, b), \zeta^{*}=\bar{\zeta}^{*}(\tau, a, b)$ of (3.2) is unique, exists for $0 \leqq \tau<\infty$, and satisfies the inequalities

$$
\left|\bar{\eta}^{*}(\tau, a, b)\right|,\left|\bar{\zeta}^{*}(\tau, a, b)\right| \leqq K\left(|a|+|b| e^{-\sigma \tau / 2}\right) \leqq \gamma_{2} \quad(0 \leqq \tau<\infty) .
$$

Furthermore, any solution $\eta^{*}(\tau), \zeta^{*}(\tau)$ of (3.2) however near $S^{\prime}(a)$ but not on $S^{\prime}(a)$ at $\tau=0$ cannot satisfy $\left|\eta^{*}(\tau)\right|,\left|\zeta^{*}(\tau)\right| \leqq \gamma_{2}$ for $\tau \geqq 0$.

The manifold $S^{\prime}(a)$ is defined by

$$
S^{\prime}(a)=\left\{\left(\begin{array}{c}
\eta^{*} \\
\zeta^{*}
\end{array}\right) \mid \eta^{*}=b, \zeta^{*}=z^{*}(a, b)\right\}
$$

where

$$
z^{*}(a, b)=-\int_{0}^{\infty} e^{-C_{0 \pi}} S\left(0, a, \bar{\eta}^{*}(\pi, a, b), \bar{\zeta}^{*}(\pi, a, b), 0\right) d \pi .
$$

The solution $\bar{y}^{*}$ of (1.6), referred to in Theorem 2 , is defined by setting $\eta^{*}=\bar{\eta}^{*}, \zeta^{*}=\bar{\zeta}^{*}$ in (3.1). Clearly Theorem 2 is an immediate consequence of this lemma.

For $a=0$ the lemma is a specialization of a well-known theorem on conditional stability, see e.g., $[1$, p. 330$]$. The general case, i.e., $a$ not necessarily zero, requires a separate proof as it is not in general true that $R(0, a, 0,0,0)$ $=0, S(0, a, 0,0,0)=0$. We shall not give this proof, however, as only rather minor changes are needed in the proof just cited for $a=0$ in order to extend it to the general case.

It is necessary for our present purposes to state that $\bar{\eta}^{*}(\tau, a, b), \bar{\zeta}^{*}(\tau, a, b)$ is the unique solution of the system of integral equations

$$
\begin{aligned}
& \eta^{*}(\tau)=e^{B_{0} \tau} b+\int_{0}^{\tau} e^{B_{0}(\tau-\pi)} R\left(0, a, \eta^{*}(\pi), \zeta^{*}(\pi), 0\right) d \pi, \\
& \zeta^{*}(\tau)=-\int_{\tau}^{\infty} e^{-C_{0}(\pi-\tau)} S\left(0, a, \eta^{*}(\pi), \zeta^{*}(\pi), 0\right) d \pi
\end{aligned}
$$

such that if $|a| \leqq \gamma_{1},|b| \leqq \gamma_{1}$ then $\left|\eta^{*}(\tau)\right|,\left|\zeta^{*}(\tau)\right| \leqq \gamma_{2}$ for $\tau \geqq 0$.

4. Proof of Theorem 3. The first inequality of (1.9) is an immediate consequence of the boundedness of $d \bar{x} / d t$ which we have mentioned earlier in connection with (1.5). In order to prove the second inequality of (1.9) we shall obtain a similar one for $\rho(t, a, b, \epsilon)=\left|\bar{\eta}(t, a, b, \epsilon)-\bar{\eta}^{*}(t / \epsilon, a, b)\right|$ $+\left|\bar{\zeta}(t, a, b, \epsilon)-\bar{\zeta}^{*}(t / \epsilon, a, b)\right|$ and then use the changes of variables (2.1) and 
(3.1). Upon setting $\tau=t / \epsilon, \pi=s / \epsilon$ and replacing $\eta^{*}, \zeta^{*}$ with $\bar{\eta}^{*}, \bar{\zeta}^{*}$ in (3.4) one obtains

$$
\bar{\eta}^{*}(t / \epsilon, a, b)=e^{B_{0} t / \epsilon} b+\frac{1}{\epsilon} \int_{0}^{t} e^{B_{0}(t-s) / \epsilon} R\left(0, a, \bar{\eta}^{*}(s / \epsilon, a, b), \bar{\zeta}^{*}(s / \epsilon, a, b), 0\right) d s
$$

$$
\xi^{*}(t / \epsilon, a, b)=-\frac{1}{\epsilon} \int_{t}^{\infty} e^{-C_{0}(s-t) / \epsilon} S\left(0, a, \bar{\eta}^{*}(s / \epsilon, a, b), \bar{\zeta}^{*}(s / \epsilon, a, b), 0\right) d s .
$$

The abbreviated notation

$$
\begin{aligned}
\bar{\xi}(t, \epsilon) & =\bar{\xi}(t, a, b, \epsilon), & \bar{\eta}(t, \epsilon)=\bar{\eta}(t, a, b, \epsilon), & \bar{\zeta}(t, \epsilon)=\bar{\zeta}(t, a, b, \epsilon) \\
\bar{\eta}^{*}(t / \epsilon) & =\bar{\eta}^{*}(t / \epsilon, a, b), & \bar{\zeta}^{*}(t / \epsilon)=\bar{\zeta}^{*}(t / \epsilon, a, b) &
\end{aligned}
$$

and $\rho(t, \epsilon)=\rho(t, a, b, \epsilon)$ will be used in the following. From (2.6), with $\bar{\xi}, \bar{\eta}, \bar{\zeta}$ replacing $\xi, \eta, \zeta$, and from (4.1) one has

$$
\begin{aligned}
& \bar{\eta}(t, \epsilon)-\bar{\eta}^{*}(t / \epsilon)=E_{1}+E_{2}+E_{3}+E_{4}, \\
& \bar{\zeta}(t, \epsilon)-\bar{\zeta}^{*}(t / \epsilon)=E_{5}+E_{6}+E_{7}+E_{8}
\end{aligned}
$$

where

$$
\begin{aligned}
E_{1}=\frac{1}{\epsilon} \int_{0}^{t} e^{B_{0}(t-s) / \epsilon}\{R(0, a, \bar{\eta}(s, \epsilon), \bar{\zeta}(s, \epsilon), 0) & \\
& \left.-R\left(0, a, \bar{\eta}^{*}(s / \epsilon), \bar{\zeta}^{*}(s / \epsilon), 0\right)\right\} d s,
\end{aligned}
$$

(4.3b) $\quad E_{2}=\frac{1}{\epsilon} \int_{0}^{t}\left\{\phi(t, s, \epsilon)-e^{B_{0}(t-s) / \epsilon}\right\} R(0, a, \bar{\eta}(s, \epsilon), \bar{\zeta}(s, \epsilon), 0) d s$,

$$
E_{3}=\frac{1}{\epsilon} \int_{0}^{t} \phi(t, s, \epsilon)\{R(s, \bar{\xi}(s, \epsilon), \bar{\eta}(s, \epsilon), \bar{\xi}(s, \epsilon), \epsilon)
$$

(4.3d) $\quad E_{4}=\left\{\phi(t, 0, \epsilon)-e^{B_{0} t / \epsilon}\right\} b$,

$$
\begin{aligned}
E_{5}=\frac{1}{\epsilon} \int_{t}^{T} e^{-C_{0}(s-t) / \epsilon}\left\{S \left(0, a, \bar{\eta}^{*}(s / \epsilon), \bar{\zeta}^{*}(s / \epsilon, 0)\right.\right. & \\
& -S(0, a, \bar{\eta}(s, \epsilon), \bar{\zeta}(s, \epsilon, 0)\} d s,
\end{aligned}
$$

(4.3f) $\quad E_{6}=\frac{1}{\epsilon} \int_{t}^{T}\left\{e^{-C_{0}(s-t) / \epsilon}-\psi(t, s, \epsilon)\right\} S(0, a, \bar{\eta}(s, \epsilon), \bar{\xi}(s, \epsilon), 0) d s$,

$(4.3 \mathrm{~g}) \quad E_{7}=\frac{1}{\epsilon} \int_{t}^{T} \psi(t, s, \epsilon)\{S(0, a, \bar{\eta}(s, \epsilon), \bar{\zeta}(s, \epsilon), 0)$

$$
-S(s, \bar{\xi}(s, \epsilon), \bar{\eta}(s, \epsilon), \bar{\zeta}(s, \epsilon), \epsilon)\} d s,
$$

(4.3h) $\quad E_{8}=\frac{1}{\epsilon} \int_{T}^{\infty} e^{-C_{0}(s-t) / \epsilon} S\left(0, a, \bar{\eta}^{*}(s / \epsilon), \bar{\zeta}^{*}(s / \epsilon), 0\right) d s$. 
The hypothesis on the characteristic roots of $B(t)$ and $C(t)$ imply the wellknown inequalities

$$
\left|e^{B_{0} t}\right|, \quad\left|e^{-C_{0} t}\right| \leqq K e^{-\sigma t} \quad(t \geqq 0)
$$

where without loss of generality we may assume that $\sigma$ is the same as in (2.5).

Let $\delta>0$, we shall define $\delta$ precisely later, then from (2.3), (4.3a), (4.3e), and (4.4) we obtain

$$
\begin{aligned}
& \left|E_{1}\right| \leqq \frac{K \delta}{\epsilon} \int_{0}^{t} e^{-\sigma(t-s) / \epsilon} \rho(s, \epsilon) d s, \\
& \left|E_{5}\right| \leqq \frac{K \delta}{\epsilon} \int_{t}^{T} e^{-\sigma(s-t) / \epsilon} \rho(s, \epsilon) d s
\end{aligned}
$$

for $0 \leqq t \leqq T, 0<\epsilon \leqq \gamma_{1}$ if $|a|,|\bar{\eta}|,\left|\bar{\eta}^{*}\right|,|\bar{\zeta}|,\left|\bar{\zeta}^{*}\right| \leqq \gamma(\delta)$. Expressing the integral in (4.5b) as the sum

$$
\frac{K \delta}{\epsilon} \int_{t}^{t+\epsilon^{1 / 2}} e^{-\sigma(s-t) / \epsilon} \rho(s, \epsilon) d s+\frac{K \delta}{\epsilon} \int_{t+\epsilon^{1 / 2}}^{T} e^{-\sigma(s-t) / \epsilon} \rho(s, \epsilon) d s
$$

and observing that (2.7) and (3.3) imply that the second of these integrals is bounded by $\omega(\epsilon)$ for $t \leqq T / 2$ and $\epsilon \leqq \min \left(\gamma_{1}, T^{2} / 4\right)$, one has

$$
\left|E_{\mathrm{b}}\right| \leqq \frac{K \delta}{\epsilon} \int_{t}^{t+\epsilon^{1 / 2}} e^{-\sigma(s-t) / \epsilon} \rho(s, \epsilon) d s+\omega(\epsilon)
$$

for $0 \leqq t \leqq T / 2,0<\epsilon \leqq \min \left(\gamma_{1}, T^{2} / 4\right)$ if $|a|,|\bar{\eta}|,\left|\bar{\eta}^{*}\right|,|\bar{\zeta}|,\left|\bar{\zeta}^{*}\right| \leqq \gamma(\delta)$.

In the following $h(t)$ will be used generically to denote a continuous function of $t$ which is defined for $t \geqq 0$ and sufficiently small and which vanishes at $t=0$.

We shall now obtain two inequalities, (4.8) and (4.12), which are similar to the inequality

$$
\left|\phi(t, s, \epsilon)-e^{B(t)(t-s) / \epsilon}\right| \leqq \omega(\boldsymbol{\epsilon}) e^{-\sigma(t-s) / \epsilon} \quad(0 \leqq s \leqq t \leqq T)
$$

obtained in [2] as a by-product of the proof of the second inequality of (2.5). The definition of $\phi(t, s, \boldsymbol{\epsilon})$ and the variation of constants formula readily imply that

$$
\phi(t, s, \epsilon)=e^{B_{0}(t-s) / \epsilon}+\frac{1}{\epsilon} \int_{s}^{t} e^{B_{0}(t-\lambda) / \epsilon}\left(B(\lambda)-B_{0}\right) \phi(\lambda, s, \epsilon) d \lambda
$$

which, together with (2.5) and (4.4), yields for $0 \leqq s \leqq t \leqq T$

$$
\begin{aligned}
\left|\phi(t, s, \epsilon)-e^{B_{0}(t-s) / \epsilon}\right| & \leqq \frac{K}{\epsilon} \int_{s}^{t} e^{-\sigma(t-\lambda) / \epsilon}\left|B(\lambda)-B_{0}\right| e^{-\sigma(\lambda-s) / 2 \epsilon} d \lambda \\
& \leqq \frac{2 K}{\sigma} \max _{0 \leqq \lambda \leqq t}\left|B(\lambda)-B_{0}\right| e^{-\sigma(t-s) / 2 \epsilon}
\end{aligned}
$$


Thus

$$
\left|\phi(t, s, \epsilon)-e^{B_{0}(t-s) / \epsilon}\right| \leqq h(t) e^{-\sigma(t-s) / 2 \epsilon} \quad(0 \leqq s \leqq t \leqq T) .
$$

From (2.7), (4.3b), and (4.8) we obtain

$$
\left|E_{2}\right| \leqq \frac{K}{\epsilon} \int_{0}^{t}\left|\phi(t, s, \epsilon)-e^{B_{0}(t-s) / \epsilon}\right| d s \leqq h(t) \quad(0 \leqq t \leqq T) .
$$

Similarly from the definition of $\psi(t, s, \epsilon)$ and the variation of constants formula one has

$$
\psi(t, s, \epsilon)=e^{-C_{0}(s-t) / \epsilon}-\frac{1}{\epsilon} \int_{t}^{8} e^{-C_{0}(\lambda-t) / \epsilon}\left(C(\lambda)-C_{0}\right) \psi(\lambda, s, \epsilon) d \lambda
$$

which, together with (2.5) and (4.4), yields for $0 \leqq t \leqq s \leqq T$

$$
\left|\psi(t, s, \epsilon)-e^{-C_{0}(s-t) / \epsilon}\right| \leqq \frac{K}{\epsilon} \int_{t}^{s} e^{-\sigma(\lambda-t) / \epsilon}\left|C(\lambda)-C_{0}\right| e^{-\sigma(\varepsilon-\lambda) / 2 \epsilon} d \lambda .
$$

Making the restriction $0 \leqq t \leqq T / 2,0<\epsilon \leqq T^{2} / 4$ in (4.11) yields

$$
\begin{aligned}
\left|\psi(t, s, \epsilon)-e^{-C_{0}(s-t) / \epsilon}\right| \leqq & \frac{K}{\epsilon} \max _{t \leqq \lambda \leqq t+\epsilon^{1 / 2}}\left|C(\lambda)-C_{0}\right| \int_{t}^{t+\epsilon / 2} e^{-\sigma(\lambda-t) / \epsilon} e^{-\sigma(s-\lambda) / 2 \epsilon} d \lambda \\
& +\frac{K}{\epsilon} \int_{t+\epsilon^{1 / 2}}^{s} e^{-\sigma(\lambda-t) / \epsilon} e^{-\sigma(s-\lambda) / 2 \epsilon} d \lambda
\end{aligned}
$$

if $t+\epsilon^{1 / 2} \leqq s$, which, together with a similar argument for $t \leqq s \leqq t+\epsilon^{1 / 2}$, implies that

$$
\left|\psi(t, s, \epsilon)-e^{-C_{0}(s-t) / \epsilon}\right| \leqq\{h(t)+\omega(\epsilon)\} e^{-\sigma(s-t) / 2 \epsilon}
$$

for $0 \leqq t \leqq T / 2,0<\epsilon \leqq T^{2} / 4, t \leqq s \leqq T$.

From (2.7), (4.3f), and (4.12) we easily obtain

$$
\left|E_{6}\right| \leqq \frac{K}{\epsilon} \int_{t}^{T}\left|\psi(t, s, \epsilon)-e^{-C_{0}(s-t) / \epsilon}\right| d s \leqq h(t)-\omega(\epsilon)
$$

for $0 \leqq t \leqq T / 2,0<\epsilon \leqq \min \left(T^{2} / 4, \gamma_{1}\right)$.

It follows from (2.5) and (4.3c) that

$$
\left|E_{3}\right| \leqq \frac{K}{\epsilon} \int_{0}^{t} e^{-\sigma(t-s) / \epsilon} \mid R(s, \bar{\xi}(s, \epsilon), \bar{\eta}(s, \epsilon), \bar{\zeta}(s, \epsilon), \epsilon)
$$

$$
-R(0, a, \bar{\eta})(s, \epsilon), \bar{\zeta}(s, \epsilon), 0) \mid d s
$$

Using the continuity of $R$ and

$$
|\bar{\xi}(s, \epsilon)-a| \leqq h(s) \quad(0 \leqq s \leqq T)
$$


which follows from (2.7) and the first equation of (2.6), we observe that the term involving $R$ in (4.14) is bounded by $h(s)+\omega(\epsilon)$. Hence

$$
\left|E_{3}\right| \leqq \frac{K}{\epsilon} \int_{0}^{t} e^{-\sigma(t-s) / \epsilon} h(s) d s+\omega(\epsilon) .
$$

If $0 \leqq t \leqq \epsilon^{1 / 2}$, then

$$
\frac{1}{\epsilon} \int_{0}^{t} e^{-\sigma(t-s) / \epsilon} h(s) d s \leqq \frac{1}{\sigma} \max _{0 \leqq s \leqq \epsilon^{1 / 2}} h(s) \leqq \omega(\epsilon) .
$$

If $0<\epsilon^{1 / 2} \leqq t$, then

$$
\begin{aligned}
\frac{1}{\epsilon} \int_{0}^{t} e^{-\sigma(t-s) / \epsilon} h(s) d s \leqq & \frac{K}{\epsilon} \int_{0}^{t-\epsilon^{1 / 2}} e^{-\sigma(t-s) / \epsilon} d s \\
& +\frac{1}{\epsilon} \int_{t-\epsilon^{1 / 2}}^{t} e^{-\sigma(t-s) / \epsilon}\{h(t)+\omega(\epsilon)\} d s \leqq h(t)+\omega(\epsilon) .
\end{aligned}
$$

Thus

$$
\left|E_{3}\right| \leqq h(t)+\omega(\epsilon) \quad\left(0 \leqq t \leqq T, 0<\epsilon \leqq \gamma_{1}\right) .
$$

Similarly it may be shown that

$$
\left|E_{7}\right| \leqq h(t)+\omega(\epsilon) \quad\left(0 \leqq t \leqq T / 2,0<\epsilon \leqq \min \left(\gamma_{1}, T^{2} / 4\right)\right) .
$$

From (4.3d) and (4.8) for $s=0$ one has

$$
\left|E_{4}\right| \leqq h(t) \quad(0 \leqq t \leqq T) .
$$

From (3.3), (4.3h), and (4.4) one has

$$
\left|E_{8}\right| \leqq \frac{K}{\epsilon} \int_{T}^{\infty} e^{-\sigma(s-t) / \epsilon} d s
$$

Hence

$$
\left|E_{8}\right| \leqq \omega(\epsilon) \quad(0 \leqq t \leqq T / 2) .
$$

It is now clear from (4.2), (4.5a, c), (4.9), (4.13), (4.16), (4.17), (4.18), and (4.19) that there exists a $t_{1}>0$ and an $\epsilon_{1}>0$, where both $t_{1}$ and $\epsilon_{1}$ are independent of $\epsilon$ and $\delta$ and where $t_{1}+\epsilon_{1}^{1 / 2} \leqq T$, such that

$$
\begin{aligned}
& \text { (4.20a) }\left|\bar{\eta}(t, \epsilon)-\bar{\eta}^{*}(t / \epsilon)\right| \leqq \frac{K \delta}{\epsilon} \int_{0}^{t} e^{-\sigma(t-s) / \epsilon} \rho(s, \epsilon) d s+h(t)+\omega(\epsilon), \\
& \text { (4.20b) }\left|\bar{\zeta}(t, \epsilon)-\bar{\zeta}^{*}(t / \epsilon)\right| \leqq \frac{K \delta}{\epsilon} \int_{t}^{t+\epsilon^{1 / 2}} e^{-\sigma(s-t) / \epsilon} \rho(s, \epsilon) d s+h(t)+\omega(\epsilon)
\end{aligned}
$$

if $0 \leqq t \leqq t_{1} ; 0<\epsilon \leqq \epsilon_{1} ;|a|,|\bar{\eta}|,\left|\bar{\eta}^{*}\right|,|\bar{\zeta}|,\left|\bar{\zeta}^{*}\right| \leqq \gamma(\delta)$. In the inequalities that follow the maxima are functions of $\epsilon$. From (4.20a) one has 


$$
\left|\bar{\eta}(t, \epsilon)-\bar{\eta}^{*}(t / \epsilon)\right| \leqq K \delta \max _{0 \leqq s \leqq t} \rho(s, \epsilon)+\max _{0 \leqq s \leqq t} h(s)+\omega(\epsilon) .
$$

The left hand side of (4.21) may now be replaced by the maximum over $0 \leqq s \leqq t$ and the right hand side increased as indicated by

$$
\begin{aligned}
\max _{0 \leq s \leq t}\left|\eta(s, \epsilon)-\bar{\eta}^{*}(s / \epsilon)\right| & \leqq K \delta \max _{0 \leq s \leq t+\epsilon^{1 / 2} \rho(s, \epsilon)} \\
& +\max _{0 \leq s \leq t} h(s)+\omega(\epsilon) .
\end{aligned}
$$

Similarly, (4.20b) yields

$$
\max _{0 \leqq s \leqq t}\left|\bar{\zeta}(s, \epsilon)-\bar{\zeta}^{*}(s / \epsilon)\right| \leqq K \delta \max _{0 \leqq s \leqq t+\epsilon^{\mathrm{N} / 2}} \rho(s, \epsilon)+\max _{0 \leqq s \leqq t} h(s)+\omega(\epsilon) .
$$

From (4.22) and (4.23) it follows that

$$
\max _{0 \leqq s \leqq t} \rho(s, \epsilon) \leqq K \delta \max _{0 \leqq s \leqq t+\epsilon^{1 / 2}} \rho(s, \epsilon)+\max _{0 \leqq s \leqq t} h(s)+\omega(\epsilon)
$$

if $0 \leqq t \leqq t_{1} ; 0<\epsilon \leqq \epsilon_{1} ;|a|,|\bar{\eta}|,\left|\bar{\eta}^{*}\right|,|\bar{\zeta}|,\left|\bar{\zeta}^{*}\right| \leqq \gamma(\delta)$.

We now define $\delta=1 / 2 K$ where $K$ is the same as in (4.24). This determines a value for $\gamma(\delta)$. From (2.7) and (3.3) it is evident that the conditions $|\bar{\eta}|$, $\left|\bar{\eta}^{*}\right|,|\bar{\zeta}|,\left|\bar{\zeta}^{*}\right| \leqq \gamma(\delta)$ may be satisfied by taking $|a|,|b|$, and $\epsilon$ sufficiently small. The $\epsilon$ part of this requirement may be satisfied by taking $\epsilon_{1}$ sufficiently small.

It may be shown by induction that for any positive integer $n$

$$
\begin{aligned}
\max _{0 \leqq s \leqq l} \rho(s, \epsilon) \leqq & \left(\frac{1}{2}\right)^{n} \max _{0 \leqq s \leqq t+n \epsilon^{1 / 2}} \rho(s, \epsilon) \\
& +\sum_{k=0}^{n-1}\left(\frac{1}{2}\right)^{k}\left\{\max _{0 \leqq s \leqq t+k \epsilon^{1 / 2}}+h(s)+\omega(\epsilon)\right\}
\end{aligned}
$$

where $h$ and $\omega$ are as in (4.24), if $0 \leqq t+(n-1) \epsilon^{1 / 2} \leqq t_{1}, 0<\epsilon \leqq \epsilon_{1}$. Note that for $n=1(4.25)$ reduces to (4.24), where $K \delta=1 / 2$. After assuming that (4.25) is true for $n=m$, it is an easy matter to show that it is true for $n=m+1$. We omit the details. From (4.25) one has

(4.26) $\max _{0 \leqq s \leqq t} \rho(s, \epsilon) \leqq\left(\frac{1}{2}\right)^{n} \max _{0 \leqq s \leqq t+n \epsilon^{1 / 2}} \rho(s, \epsilon)+2\left\{\max _{0 \leqq s \leqq t+(n-1) \epsilon^{1 / 2}} h(s)+\omega(\epsilon)\right\}$ if $0 \leqq t+(n-1) \epsilon^{1 / 2} \leqq t_{1}, 0<\epsilon \leqq \epsilon_{1}$.

Let $\tilde{\alpha}>0$, as in the statement of Theorem 3. Let $n_{\tilde{\alpha}}$ be the smallest positive integer such that $\left(\frac{1}{2}\right)_{\tilde{\alpha}}^{n} \leqq \tilde{\alpha} / 5 K$, where $K$ satisfies $\rho(t, \epsilon) \leqq K$ for $0 \leqq t \leqq T$, $0<\epsilon \leqq \gamma_{1}$. From the continuity of $\omega(\epsilon)$ and $h(t)$ there exist $\beta_{1}(\tilde{\alpha})>0$ and $\beta_{2}(\tilde{\alpha})>0$ such that $0 \leqq \omega(\epsilon) \leqq \tilde{\alpha} / 5$ if $0<\epsilon \leqq \beta_{1}$ and $0 \leqq h(t) \leqq \tilde{\alpha} / 5$ if $0 \leqq t \leqq \beta_{2}$. Define $\tilde{\boldsymbol{\beta}}(\tilde{\boldsymbol{\alpha}})$ by 


$$
\tilde{\beta}(\tilde{\alpha})=\min \left\{\frac{t_{1}}{2}, \frac{\beta_{2}}{2}, \frac{t_{1}^{2}}{4 n_{\sim}^{2}}, \frac{\beta_{2}^{2}}{4 n_{\tilde{\alpha}}^{2}}, \epsilon_{1}, \beta_{1}\right\} .
$$

It then follows from (4.26) that

$$
\rho(t, \epsilon) \leqq \tilde{\alpha}
$$

(if $0 \leqq t \leqq \tilde{\beta}(\tilde{\alpha}), 0<\epsilon \leqq \tilde{\beta}(\tilde{\alpha})$ ).

However, from (2.1), (2.7), (3.1), (3.3), (4.15), and the definition of $\rho(t, \epsilon)$ one easily obtains

$$
\left|\bar{y}(t, a, b, \epsilon)-\bar{y}^{*}(t / \epsilon, a, b)\right| \leqq h(t)+K \rho(t, \epsilon) .
$$

The conclusion of Theorem 3 is obvious from (4.27) and (4.28).

\section{BIBLIOGRAPHY} 1955.

1. E. A. Coddington and N. Levinson, Theory of ordinary differential equations, New York,

2. L. Flatto and N. Levinson, Periodic solutions of singularly perturbed systems, Journal of Rational Mechanics and Analysis vol. 4 (1955) pp. 943-950.

3. K. O. Friedrichs and W. R. Wasow, Singular perturbation of non-linear oscillations, Duke Math. J. vol. 13 (1946) pp. 367-381.

4. I. S. Gradstein, On a class of non-linear differential equations with small coefficients for certain derivatives, Doklady Akademii Nauk SSSR. (N.S.) vol. 64 (1949) pp. 441-443.

5. J. J. Levin, On singular perturbations of nonlinear systems of differential equations related to conditional stability, Duke Math. J. vol. 24 (1956) pp. 609-620.

6. J. J. Levin and N. Levinson, Singular perturbations of non-linear systems of differential equations and an associated boundary layer equation, Journal of Rational Mechanics and Analysis vol. 3 (1954) pp. 247-270.

7. A. Tihonov, On systems of differential equations containing parameters, Rec. Math. (Mat. Sbornik) N.S. vol. 27 (69) (1950) pp. 147-156.

Massachusetts Institute of Technology,

Cambridge, Mass. 